HEAD AND NECK

\title{
VITOM-3D-assisted retroauricular neck surgery (RANS-3D): preliminary experience at Candiolo Cancer Institute
}

\author{
Chirurgia cervicale VITOM-3D assistita con accesso retroauricolare (RANS-3D): \\ l'esperienza preliminare dell'Istituto di Candiolo
}

\author{
Erika Crosetti ${ }^{1}$, Giulia Arrigoni ${ }^{1}$, Alessandra Caracciolo ${ }^{1}$, Martina Tascone ${ }^{1}$, Andrea Manca ${ }^{1}$, Giovanni Succo ${ }^{1,2}$ \\ ${ }^{1}$ Head and Neck Oncology Service, Candiolo Cancer Institute, FPO - IRCCS, Candiolo (TO), Italy; ${ }^{2}$ Department of Oncology, \\ University of Turin, Orbassano (TO), Italy
}

\section{SUMMARY}

Objective. The recent introduction of 3D exoscopic surgery has engendered interesting technical improvements in head and neck surgery. The main goal of this study was to describe the application of 3D exoscopic technology on a wide range of pathologies of the neck, benign and malignant, through a minimally invasive retroauricular approach.

Methods. In the period January-December, 2019, 40 consecutive patients underwent neck surgery with a retroauricular approach, enhanced by using a 3D exoscope at the Head and Neck Oncological Unit of Candiolo Cancer Institute.

Results. Data regarding time to drain removal, length of hospitalisation, degree of pain experienced, need for opioid drugs during hospitalisation and after discharge, and intraoperative and post-operative complications were collected. All patients were followed for a minimum of 90 days with possible complications evaluated at each post-operative visit. Post-operative outcomes were evaluated at 3 months after surgery.

Conclusions. The current study indicates that VITOM-3D-assisted retroauricular neck surgery (RANS-3D) may be an interesting approach for neck surgery. The hybrid execution of neck dissection under direct and exoscopic vision represents a valid alternative to videoassisted endoscopic- and robot-assisted techniques.

KEY WORDS: 3D, neck dissection, RANS, exoscopic surgery

\section{RIASSUNTO}

Oggetto. Il recente avvento della chirurgia esoscopica $3 D$ ha consentito, nell'ambito della chirurgia cervico-cefalica, l'introduzione di interessanti innovazioni tecnologiche. L'obiettivo del seguente studio è stato quello di descrivere l'impiego della tecnologia esoscopica $3 D$ su un'ampia gamma di patologie cervico-cefaliche, benigne e maligne, trattate con accesso mini-invasivo retroauricolare.

Metodi. Nel periodo Gennaio-Dicembre 2019 presso la Divisione di Chirurgia Oncologica Cervico-Cefalica dell'Istituto IRCCS-FPO di Candiolo 40 pazienti consecutivi sono stati sottoposti a chirurgia cervicale con incisione retroauricolare con ausilio dell'esoscopio $3 D$.

Risultati. Sono stati raccolti $i$ dati relativi alla durata di mantenimento del drenaggio, durata della degenza ospedaliera, entità del dolore lamentato dal paziente (scala VAS), necessità di somministrazione di oppioidi durante la degenza e dopo la dimissione, complicanze intra- e postoperatorie. Tutti i pazienti sono stati seguiti per un periodo minimo di 90 giorni, valutando, ad ogni visita post-operatoria, la comparsa di possibili complicanze. I risultati post-operatori sono stati valutati a distanza di 3 mesi dall'intervento chirurgico. Conclusioni. Il seguente studio conferma che il RANS-3D rappresenta un interessante approccio chirurgico al collo. L'esecuzione ibrida della dissezione laterocervicale, sotto visione diretta ed esoscopica, rappresenta una valida alternativa alle tecniche endoscopiche e robot-assistite.

PAROLE CHIAVE: 3D, dissezione del collo, RANS, chirurgia esoscopica
Received: December 3, 2020

Accepted: March 10, 2021

\section{Correspondence}

Erika Crosetti

Head and Neck Oncology Unit, FPO IRCCS, Candiolo Cancer Institute, 10060 Candiolo - Turin, Italy Tel.+ 390119933663

E-mail: erika.crosetti@ircc.it

\section{Funding}

This research was funded by Regione Piemonte $\mathrm{AD}$ FUNCTIONEM (years 2019-2021); FPRC 5x1000 2016 Ministero della Salute Progetto ARDITE; Fondi Ricerca Corrente 2020, Ministero della Salute.

Conflict of interest

The Authors declare no conflict of interest.

How to cite this article: Crosetti E, Arrigoni G, Caracciolo A, et al. VITOM-3D-assisted retroauricular neck surgery (RANS-3D): preliminary experience at Candiolo Cancer Institute. Acta Otorhinolaryngol Ital 2021;41:419-431. https:// doi.org/10.14639/0392-100X-N1293

๑ Società Italiana di Otorinolaringoiatria e Chirurgia Cervico-Facciale

\section{(c) (1) $\Theta$}

This is an open access article distributed in accordance with the CC-BY-NC-ND (Creative Commons Attribution-NonCommercial-NoDerivatives 4.0 International) license. The article can be used by giving appropriate credit and mentioning the license, but only for non-commercial purposes and only in the original version. For further information: https:// creativecommons.org/licenses/by-nc-nd/4.0/deed.en 


\section{Introduction}

Traditionally, the surgical treatment of head and neck pathologies involves open neck approaches, giving the surgeon a direct domain of the disease. However, this can sometimes result in negative post-operative aesthetic-functional sequelae.

As far as the treatment of neck pathologies is concerned, to date, not much has changed regarding surgical technique, and the open neck approach remains the most frequently used method ${ }^{1,2}$. On the other hand, efforts to reduce the morbidity and weight of surgical treatments have encouraged the development of minimally-invasive techniques such as transoral/transnasal endoscopic and robotic approaches to the aerodigestive tract rather than to the neck ${ }^{1,2}$. Endoscopic-assisted and robot-assisted neck dissections have been successfully described and proposed; however, they have limitations and disadvantages ${ }^{3}$. The main ones are the duration of surgery, which is longer than with the standard approach, the need for surgeons to develop skills in the field of endoscopic and robotic techniques, and the overall higher costs of the procedure ${ }^{4}$.

The recent introduction of 3D exoscopic surgery has allowed interesting improvements in head and neck surgery, with technical solutions also applicable to neck dissection, with the aim of replacing robotic surgery and minimising the costs of the procedure. In an earlier paper, a preclinical study on cadavers, our team described the principles of exoscopic-assisted neck dissection via a retroauricular approach, demonstrating its advantages and limitations ${ }^{5}$.

The main goal of this study was to describe the application of 3D exoscopic technology on a wide range of pathologies of the neck, benign and malignant, through a minimallyinvasive retroauricular approach.

\section{Materials and methods}

Forty consecutive patients underwent neck dissection by a retroauricular approach for different head and neck pathologies, benign and malignant, with visualisation enhanced using a 3D exoscope (Vitom 3D; Karl Storz, Tuttlingen, Germany). All patients were treated at the Head and Neck Oncological Unit of the FPO IRCCS, Candiolo Cancer Institute, in the period January-December, 2019.

The study received approval from the Committee of Ethics in Research in our hospital. All of the procedures were considered to be conventional in terms of technique and indications, in accordance with current guidelines and therefore in accordance with the ethical standards of the Institutional and/or National Research Committee and with the 1964 Helsinki Declaration and its later amendments. The advantages and disadvantages of this approach as well as the alternative approaches were clearly and fully explained to patients when seeking informed consent for the procedure. The inclusion criteria were as follows: patients with benign neck pathologies; patients with oral cavity/oropharynx/supraglottic larynx proven squamous cell carcinoma with or without clinically metastatic lymph nodes, staged cN0/N1 and who were candidates for elective neck dissection; patients with thyroid malignant tumours with clinically metastatic lymph nodes, staged cN1b.

All patients underwent the same clinical assessment during the 3 weeks before surgery including: clinical examination, nutritional status evaluation, biopsy/fine needle aspiration biopsy (FNAB) with p16 protein expression on biopsy in the case of malignant tumours, maxillofacial and neck MRI/CT scan and endocrinological tests. Two surgeons (G.S. and E.C.) carried out all of the procedures. The exoscope was mounted on a couple of versatile self-supporting arms, specially developed for use with VITOM ${ }^{\circledR}$ allowing straightforward and precise positioning of the system (Fig. 1).

3D-HD imaging provided a realistic sense of depth to improve anatomic orientation. In order to carry out a hybrid procedure, a large part of the operation was performed under direct visualisation and only dissection of the most distant levels/region from the incision (levels IV and I) was performed under exoscopic 3D vision. The polarising lenses were adapted to the $3.5 \times$ magnification operating loupes.

\section{Operating room setting}

The first and second surgeons sat on the same side as the neck dissection. A 55-inch monitor was positioned contralaterally in front of them, at a distance of about 2.5 metres. The assistant was placed at the head of the patient.

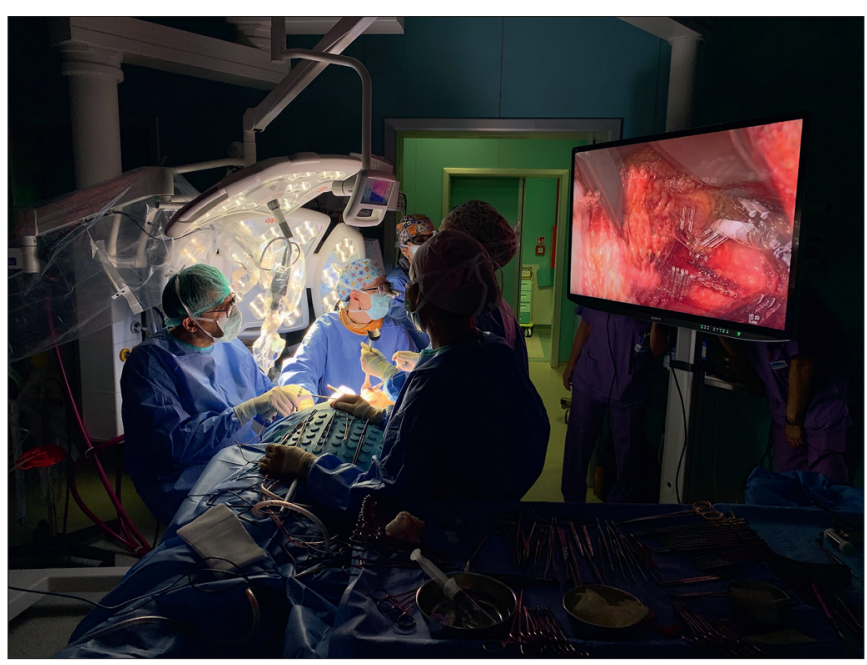

Figure 1. Typical operating room setting for VITOM-3D assisted neck dissection 
The endoscopic cart was positioned at the bottom of the operating bed with a second 3D monitor for the assistant. The first and second surgeons also wore polarising clip-on lenses over operating loupes to view the monitor when necessary. In most procedures, the exoscope was mounted on a mechanical holder, positioned behind the two surgeons and oriented towards the surgical field and was moved by an assistant. The latter controlled the exoscope by a control joystick. In some cases, a latest generation robotic holder for VITOM (ARTIP CRUISE ${ }^{\circledR}$ ) was used successfully. All team members wore 3D glasses.

Patient preparation for surgery was the same as that which is typically used for other neck surgical procedures performed under general anaesthesia. The patient was positioned on the operating table in the supine position without any interscapular support but with contralateral head rotation. It is suggestable to identify and draw the external jugular vein, if present, the margins of the platysma muscle and the sternocleidomastoid muscle.

A retroauricular incision was made, raising the subplatysmal skin flap to expose the surgical field. After elevation of the skin flap along a subplatysmal plane, a self-retaining retractor was placed (Fig. 2A, B). At this point, the dissection proceeded with the aid of the $3 \mathrm{D}$ exoscope and the two surgeons worked next to each other.
All operations were performed following conventional surgical techniques, using vascular clips and haemostatic cutting/coagulation devices such as bipolar scissors and LigaSure (LigaSure ${ }^{\mathrm{TM}}$ Small Jaw Open Sealer/Divider LF1212, Covidien, Medtronic, Minneapolis, MN, USA). Surgical instruments (forceps, scissors) had a minimum length of $24 \mathrm{~cm}$ in order to reach deep and narrow spaces without any difficulty.

Closed aspiration drains (Blake, Ethicon Inc., Somerville, NJ, USA) were placed in all cases. Patients were discharged after our clinical routine for similar surgical procedures.

The following data were collected: number of lymph nodes retrieved in sample, time to drain removal, which was carried out when the drain output was less than $20 \mathrm{~mL} / \mathrm{day}$, duration of hospitalisation, degree of pain experienced (NRS: numerical rating scale) ${ }^{6}$, need for opioid drugs during hospitalisation and after discharge, intra-operative and post-operative complications.

All patients were followed at the institute for a minimum of 90 days. At each post-operative visit, the surgeon evaluated patients for possible complications such as seroma, haematoma, surgical site infection, cranial nerve impairment, and skin flap dehiscence or necrosis. The frequency of these visits varied according to the surgical procedure carried out and the patient's specific requirements.

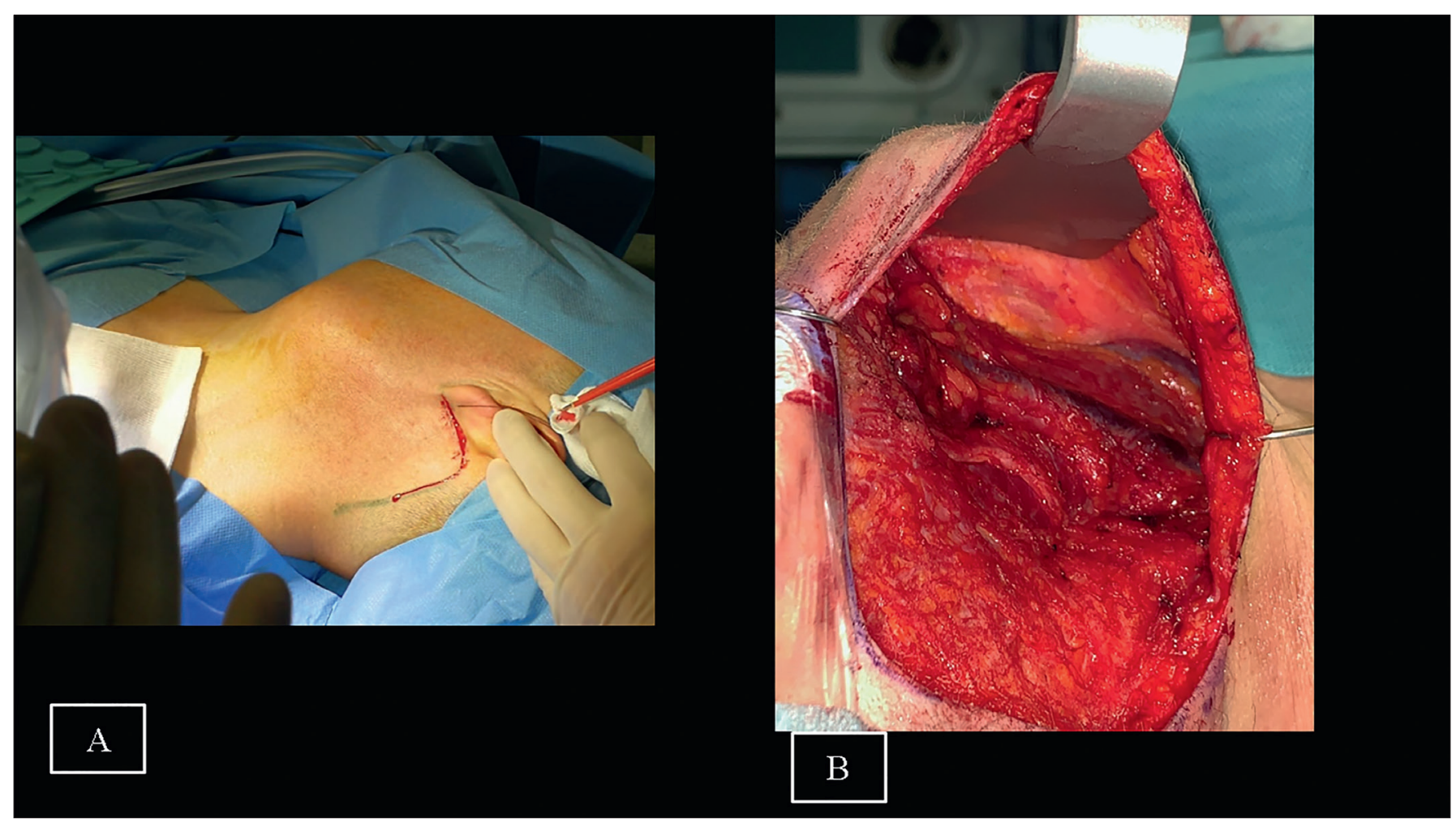

Figure 2. (A) Retroauricular skin incision. (B) Elevation of skin flap along a subplatysmal plane and placement of self-retaining retractor. 
Post-operative outcomes (satisfaction score) were evaluated at 3 months after surgery and ranged from 1 to 5 (1, extremely dissatisfied; 2, dissatisfied; 3 , average; 4, satisfied; 5 , extremely satisfied ${ }^{7}$.

Moreover, the following were evaluated: the surgeon's subjective perception of compartment-orientation, dissection and technical feasibility of the procedure (surgeon's satisfaction score, ranged from 1, dissatisfied; 2, average; 3 satisfied) and possible side effects due to the necessity to wear 3D glasses for a prolonged period.

\section{Results}

The present case series included 40 patients treated at the Head and Neck Oncological Unit of FPO-IRCCS, Candiolo Cancer Institute between January and December, 2019.
Group with benign pathology

There were 14 patients in this group (10 female, 4 male) with a median age of 43.2 years (range 16-67) (Tab. I). The average (body mass index (BMI) was 23.8, with a maximum of 32. No patients in this group experienced intra-operative or post-operative complications. Post-surgical pain was evaluated with a numerical rating scale (NRS, range $0-10$ where 0 is no pain and 10 is the worst pain imaginable $)^{6}$. Mean and peak values were 1.07 and 3 , respectively. There were no other post-operative neurologic deficits (marginalis mandibulae nerve paralysis, facial nerve paralysis, shoulder pain syndrome).

The average docking time to obtain an adequate operative setting was 8 minutes and the average intra-operative time was 68.7 minutes (22-126 minutes) (including flap harvesting, placement of retractor, dissection).

Table I. Group with benign head-neck pathologies.

\begin{tabular}{|c|c|c|c|c|c|c|c|c|c|c|c|}
\hline No. & I.D. & Sex & $\begin{array}{c}\text { Age } \\
\text { (years) }\end{array}$ & Pathology & BMI & $\begin{array}{c}\text { Duration } \\
\text { of } \\
\text { operation } \\
\text { (minutes) }\end{array}$ & $\begin{array}{l}\text { Drainage } \\
\text { period } \\
\text { (days) }\end{array}$ & $\begin{array}{l}\text { Hospital } \\
\text { stay } \\
\text { (days) }\end{array}$ & $\begin{array}{l}\text { Post- } \\
\text { operative } \\
\text { pain }^{*} \\
\text { (NRS) }\end{array}$ & Complications & $\begin{array}{l}\text { Satisfaction } \\
\text { score }^{\dagger}\end{array}$ \\
\hline 2 & C.B. & Male & 65 & Branchial cyst & 28 & 80 & 3 & 3 & 1 & None & 4 \\
\hline 3 & M.M.C. & Female & 65 & $\begin{array}{c}\text { Hyperplastic } \\
\text { cervical lymph } \\
\text { node }\end{array}$ & 32 & 40 & 2 & 2 & 0 & None & 5 \\
\hline 4 & M.G. & Male & 45 & Branchial cyst & 21 & 90 & 2 & 3 & 2 & None & 5 \\
\hline 5 & O.A. & Female & 25 & $\begin{array}{c}\text { Cystic } \\
\text { lymphangioma }\end{array}$ & 16 & 70 & 2 & 3 & 2 & None & 5 \\
\hline 6 & C.S. & Female & 25 & $\begin{array}{l}\text { Pleiomorphic } \\
\text { adenoma } \\
\text { parapharyngeal } \\
\text { space }\end{array}$ & 19 & 100 & 2 & 2 & 1 & None & 4 \\
\hline 9 & D.P.A. & Male & 55 & Lipoma & 29 & 22 & 1 & 2 & 0 & None & 5 \\
\hline 10 & S.M.R. & Female & 67 & $\begin{array}{l}\text { Parapharyngeal } \\
\text { space } \\
\text { Lymphangioma }\end{array}$ & 26 & 89 & 2 & 4 & 0 & None & 5 \\
\hline 11 & M. A. & Female & 43 & $\begin{array}{l}\text { Pleiomorphic } \\
\text { adenoma } \\
\text { parotid gland }\end{array}$ & 20 & 53 & 2 & 2 & 0 & None & 5 \\
\hline 12 & M.C. & Female & 32 & $\begin{array}{l}\text { Pleiomorphic } \\
\text { adenoma } \\
\text { Parotid gland }\end{array}$ & 19 & 45 & 2 & 2 & 0 & None & 5 \\
\hline 13 & G.M.R. & Female & 48 & $\begin{array}{l}\text { Pleiomorphic } \\
\text { adenoma } \\
\text { Parotid gland }\end{array}$ & 19 & 40 & 2 & 2 & 0 & None & 5 \\
\hline
\end{tabular}

"NRS numerical rating scale where 0 is no pain and 10 is the worst pain imaginable. ${ }^{+}$Satisfaction score: 1, extremely dissatisfied; 2, dissatisfied; 3, average; 4, satisfied; 5, extremely satisfied. 


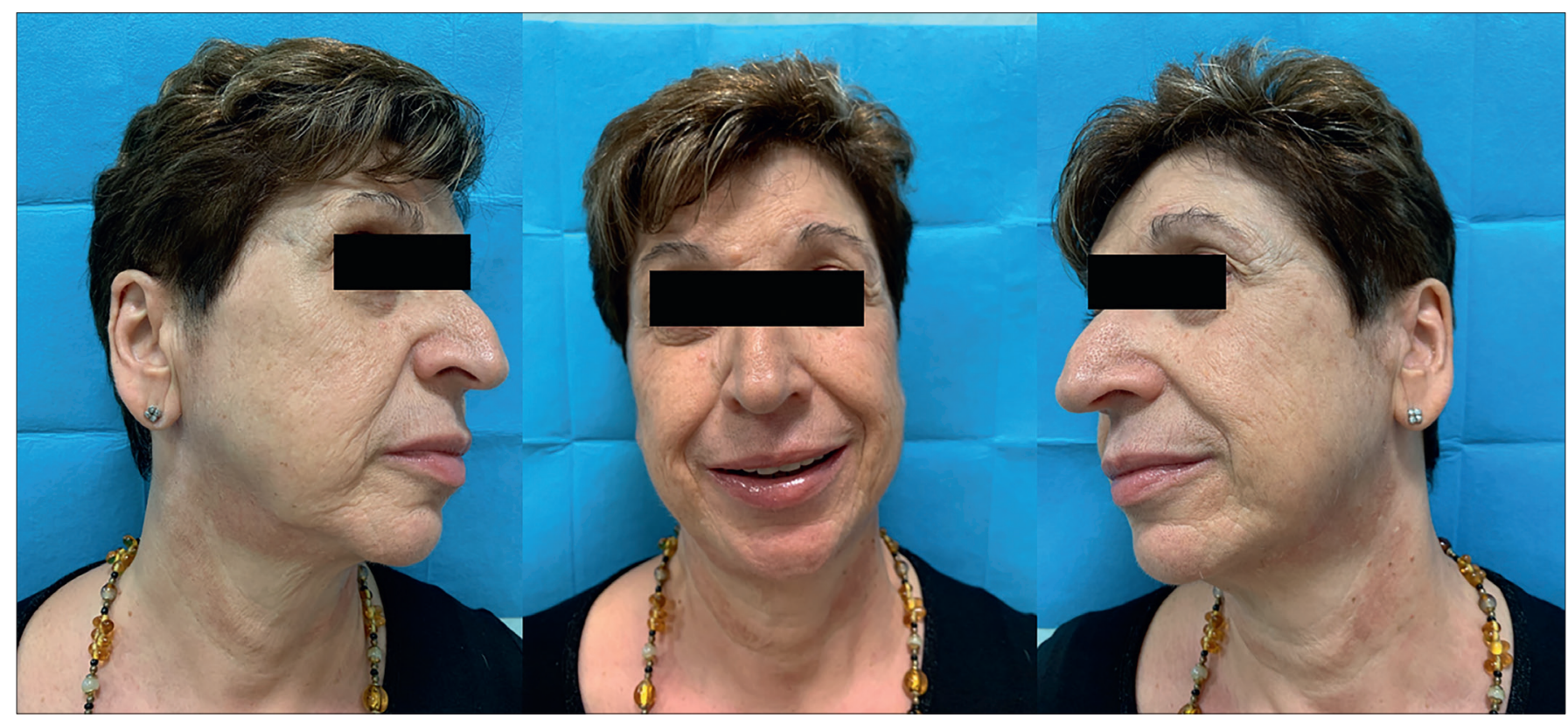

Figure 3. Cosmetic results in a patient treated for benign pathology (branchial cyst).

The average duration of drainage was 2.1 days and the average length of hospitalisation was 2.5 days. All patients were extremely satisfied with their cosmetic outcome (median satisfaction score 4.7 of 5) (Fig. 3). Both surgeons were extremely satisfied with the outcomes (median surgeon satisfaction score was 5). None of the surgeons experienced side effects. In this group of patients, the exoscope was particularly useful to the first surgeon to improve the identification of the terminal branches of the facial nerve, and to the second surgeon during haemostatic manoeuvres.

\section{Group with head-neck malignancy}

There were 16 patients in this group (4 women, 12 men), with a median age of 57.68 years (range 26-78) (Tab. II). The average BMI was 25.3 , with a maximum of 35 . Two patients were pre-treated $(12.5 \%)$ (radiotherapy for non-Hodgkin lymphoma and partial glossectomy + ipsilateral neck dissection). Both surgical procedures (on $\mathrm{T}$ and on $\mathrm{N}$ ) were carried out on the same day. A surgical tracheostomy was performed in 12 patients. The same cervical skin incision was used to dissect the lower part of level IV upwards. This surgical choice was extremely useful to facilitate the dissection of level IV, and to dramatically reduce the risk of vascular/lymphatic injury.

The concept was to create a service skin window, allowing the lateral border of the sternocleidomastoid muscle, the upper belly of the omohyoid muscle, and the cervical vascular-nervous axis to be identified and then dissecting this level upward, reaching level III. No patient needed a blood transfusion.
There were no intra-operative complications in patients in this group. A large skin flap necrosis was observed in only one patient $(6.25 \%)$, pre-treated with radiation therapy, and this was treated with a dressing. Post-surgical pain was evaluated with a NRS (range 0-10) ${ }^{6}$. Mean and peak values were 1.4 and 5, respectively. All patients received $1 \mathrm{~g}$ paracetamol intravenously three times a day to relieve pain until discharge. None needed supplemental therapy with NSAIDs or opioids.

The number of resected lymph nodes ranged from 15 to 53, with a mean of 24.7 nodes.

The average docking time to obtain an adequate operative setting was 7.5 minutes and the average intra-operative period was 97.93 minutes (38-163 minutes) (including flap harvesting, retractor placement, neck dissection).

The average duration of drainage was 3.33 days and the average length of hospitalisation was 9.625 days. One patient (6.25\%) experienced shoulder syndrome.

Post-operative radiotherapy was indicated in 6 patients showing single / multiple lymph node metastasis on the specimen without extranodal extension; concurrent chemoradiation was performed in 6 cases with extranodal extension and in 1 case staged pT4aN0. Chemotherapy alone was performed in one patient affected by non-Hodgkin lymphoma of the parotid gland. One patient did not undergo adjuvant therapy (patient \#15). In patient \#8, only one positive lymph node was found (size $4 \mathrm{~mm}$ ); after multidisciplinary discussion, a proposal for frequent clinical and radiological frequent follow-up was adopted. 
Table II. Group with head-neck malignancies (continues on page 425).

\begin{tabular}{|c|c|c|c|c|c|c|c|c|c|c|}
\hline No. & ID & $\begin{array}{c}\text { Age } \\
(y)\end{array}$ & Sex & BMI & $\begin{array}{l}\text { Pre- } \\
\text { treatment }\end{array}$ & Histology & Site & Surgery & $\begin{array}{l}\text { cTNM } \\
\text { (TNM VIII } \\
\text { Eds) }\end{array}$ & $\begin{array}{c}\text { pTNM } \\
\text { (TNM VIII Eds) }\end{array}$ \\
\hline 1 & F.R. & 53 & Male & 22 & None & SCC & $\begin{array}{l}\text { Oropharynx } \\
\text { (tonsil) }\end{array}$ & $\begin{array}{c}\text { Oropharyngectomy by TORS+ } \\
\text { ipsilateral SND } \\
\text { (II-IV levels) + tracheostomy }\end{array}$ & T3N1 & $\begin{array}{l}\text { T2N1 } \\
\text { p16+ }\end{array}$ \\
\hline 2 & M.A. & 57 & Male & 20 & None & SCC & $\begin{array}{l}\text { Oropharynx } \\
\text { (tonsil) }\end{array}$ & $\begin{array}{c}\text { Oropharyngectomy by TORS+ } \\
\text { ipsilateral SND } \\
\text { (II-IV levels) + tracheostomy }\end{array}$ & T1N0 & $\begin{array}{l}\text { T1N3b } \\
\text { p16- }\end{array}$ \\
\hline 3 & B.S. & 78 & Male & 29 & None & SCC & $\begin{array}{l}\text { Oropharynx } \\
\text { (tonsil) }\end{array}$ & $\begin{array}{c}\text { Oropharyngectomy by TORS+ } \\
\text { ipsilateral SND } \\
\text { (II-IV levels) + tracheostomy }\end{array}$ & T1N1 & $\begin{array}{l}\text { T1N1 } \\
\text { p16+ }\end{array}$ \\
\hline 4 & F.G.C. & 77 & Male & 23 & None & SCC & $\begin{array}{l}\text { Oropharynx } \\
\text { (tonsil) }\end{array}$ & $\begin{array}{c}\text { Oropharyngectomy by TORS+ } \\
\text { ipsilateral SND } \\
\text { (II-IV levels) + tracheostomy }\end{array}$ & T1N1 & $\begin{array}{l}\text { T2N1 } \\
\text { p16+ }\end{array}$ \\
\hline 5 & B.S. & 55 & Male & 24 & None & SCC & $\begin{array}{l}\text { Oropharynx } \\
\text { (tonsil) }\end{array}$ & $\begin{array}{c}\text { Oropharyngectomy by TORS+ } \\
\text { ipsilateral SND } \\
\text { (II-IV levels) + tracheostomy }\end{array}$ & T1N1 & $\begin{array}{l}\text { T1N3b } \\
\text { p16- }\end{array}$ \\
\hline 6 & M.S. & 63 & Male & 24 & None & SCC & $\begin{array}{l}\text { Oropharynx } \\
\text { (tonsil) }\end{array}$ & $\begin{array}{c}\text { Oropharyngectomy by TORS+ } \\
\text { ipsilateral SND } \\
\text { (II-IV levels) + tracheostomy }\end{array}$ & T1N1 & $\begin{array}{l}\text { T2N1 } \\
\text { p16+ }\end{array}$ \\
\hline 7 & P.I. & 59 & Male & 27 & None & SCC & $\begin{array}{l}\text { Oropharynx } \\
\text { (tonsil) }\end{array}$ & $\begin{array}{l}\text { BOT resection by TORS+ ipsilateral } \\
\text { SND (II-IV levels) + tracheostomy }\end{array}$ & TxN1 & $\begin{array}{l}\text { T1N1 } \\
\text { p16+ }\end{array}$ \\
\hline 8 & I.L. & 63 & Female & 23 & None & SCC & $\begin{array}{l}\text { Larynx } \\
\text { (supraglottis) }\end{array}$ & $\begin{array}{l}\text { Type II SPL by TORS+ bilateral } \\
\text { SND (II-IV levels) + tracheostomy }\end{array}$ & T2N1 & $\begin{array}{l}\text { T2N1 } \\
\text { p16+ }\end{array}$ \\
\hline 9 & L.A. & 26 & Male & 26 & None & $\begin{array}{l}\text { Acinic cell } \\
\text { carcinoma }\end{array}$ & $\begin{array}{l}\text { Oral cavity } \\
\text { (hard palate) }\end{array}$ & $\begin{array}{l}\text { Hard palate resection + ipsilateral } \\
\text { SND } \\
\text { (II-IV levels) + tracheostomy }\end{array}$ & T4aNO & T4aNO \\
\hline 10 & F.R. & 47 & Female & 32 & None & SCC & $\begin{array}{l}\text { Oral cavity } \\
\text { (mobile tongue) }\end{array}$ & $\begin{array}{l}\text { Marginal glossectomy + ipsilateral } \\
\text { SND } \\
(\text { I-III levels })+\text { tracheostomy }\end{array}$ & T1N0 & T1N1 \\
\hline 11 & M.I. & 68 & Female & 35 & None & SCC & CUP & $\begin{array}{c}\text { Panendoscopy + ipsilateral SND } \\
\text { (II-V levels) }\end{array}$ & TxN2 & $\begin{array}{l}\text { TxN2 } \\
\text { p16+ }\end{array}$ \\
\hline 12 & R.D. & 63 & Male & 22 & RT for LNH & SCC & $\begin{array}{l}\text { Oral cavity } \\
\text { (mobile tongue) }\end{array}$ & $\begin{array}{c}\text { Marginal glossectomy + ipsilateral } \\
\text { SND } \\
\text { (I-IV levels) + tracheostomy }\end{array}$ & T1N1 & T1N1 \\
\hline 13 & E.S. & 64 & Male & 22 & None & SCC & $\begin{array}{l}\text { Oral cavity } \\
\text { (mobile tongue) }\end{array}$ & $\begin{array}{l}\text { Marginal glossectomy + ipsilateral } \\
\text { SND } \\
\text { (I-IV levels) + tracheostomy }\end{array}$ & T1N1 & T2N3b \\
\hline 14 & C.A. & 65 & Male & 25 & None & SCC & CUP & $\begin{array}{l}\text { BOT mucosectomy + ipsilateral } \\
\text { SND (II-IV levels) }\end{array}$ & TxN1 & $\begin{array}{l}\text { TxN1 } \\
\text { p16+ }\end{array}$ \\
\hline 15 & N.M. & 52 & Female & 19 & Surgery & SCC & $\begin{array}{l}\text { Oral cavity } \\
\text { (mobile tongue }\end{array}$ & $\begin{array}{c}\text { Partial glossectomy + ipsilateral } \\
\text { SND } \\
\text { (I-IV levels) }\end{array}$ & T1N0 & T1No \\
\hline 16 & P.A. & 33 & Male & 33 & None & LNH & Parotid gland & Type II parotidectomy & & - \\
\hline
\end{tabular}

Gray boxes: pts subjected to oropharyngectomy by transoral robotic surgery (TORS) and SND by RAND-3D; BOT: base of tongue; CUP: cancer of unknown primary origin; CRT: chemoradiotherapy; RT: radiotherapy; ECS: extracapsular spread; NED: no evidence of disease; SCC: squamous cell carcinoma; SND: selective neck dissection; LNH: Non-Hodgkin lymphoma. "NRS numerical rating scale where 0 is no pain and 10 is the worst pain imaginable;

+ Satisfaction score: 1, extremely dissatisfied; 2, dissatisfied; 3, average; 4, satisfied; 5, extremely satisfied. 
Table II. Group with head-neck malignancies (follows from page 424).

\begin{tabular}{|c|c|c|c|c|c|c|c|c|c|c|}
\hline No & ID & $\begin{array}{l}\text { Lymph node } \\
\text { (positive/total) } \\
\text { (ECS, Level, } \\
\emptyset \text { max mm) }\end{array}$ & $\begin{array}{l}\text { Duration of } \\
\text { operation } \\
\text { (minutes) }\end{array}$ & $\begin{array}{l}\text { Drainage } \\
\text { period } \\
\text { (days) }\end{array}$ & $\begin{array}{l}\text { Hospitalisation } \\
\text { stay } \\
\text { (days) }\end{array}$ & $\begin{array}{l}\text { Post- } \\
\text { operative } \\
\text { pain* } \\
\text { (NRS) }\end{array}$ & Complications & $\begin{array}{l}\text { Satisfaction } \\
\text { score }^{\dagger}\end{array}$ & $\begin{array}{l}\text { Adjuvant } \\
\text { treatment }\end{array}$ & $\begin{array}{l}\text { Follow- } \\
\text { up }\end{array}$ \\
\hline 1 & F.R. & $\begin{array}{c}1 / 28 \\
\text { ECS+ Level III } \\
32 \mathrm{~mm}\end{array}$ & $\begin{array}{c}132 \\
\text { (112 RAND-3D) }\end{array}$ & 3 & 10 & 5 & None & 5 & CRT & NED \\
\hline 2 & M.A. & $\begin{array}{c}5 / 19 \\
4 \text { ECS+ level II } 14 \mathrm{~mm} \\
1 \text { ECS- level III }<1 \mathrm{~mm}\end{array}$ & $\begin{array}{c}156 \\
\text { (111 RAND-3D) }\end{array}$ & 2 & 20 & 2 & None & 5 & CRT & NED \\
\hline 3 & B.S. & $\begin{array}{c}4 / 23 \\
\text { ECS- level II-III } \\
28 \mathrm{~mm}\end{array}$ & $\begin{array}{c}153 \\
\text { (122 RAND-3D) }\end{array}$ & 3 & 10 & 1 & None & 5 & RT & NED \\
\hline 4 & F.G.C. & $\begin{array}{c}1 / 29 \\
\text { ECS- Level lla } \\
42 \mathrm{~mm}\end{array}$ & $\begin{array}{c}112 \\
\text { (58 RAND-3D) }\end{array}$ & 3 & 12 & 0 & None & 5 & RT & NED \\
\hline 5 & B.S. & $\begin{array}{c}2 / 15 \\
1 \text { ECS+ level Ila } 28 \text { mm } \\
1 \text { ECS- level III } 5 \text { mm }\end{array}$ & $\begin{array}{c}117 \\
\text { (95 RAND-3D) }\end{array}$ & 5 & 8 & 1 & None & 5 & CRT & NED \\
\hline 6 & M.S & $\begin{array}{l}3 / 17 \\
\text { ECS- level II-III } \\
30 \mathrm{~mm}\end{array}$ & $\begin{array}{c}118 \\
\text { (92 RAND-3D) }\end{array}$ & 3 & 10 & 1 & $\begin{array}{l}\text { Shoulder } \\
\text { syndrome }\end{array}$ & 5 & RT & NED \\
\hline 7 & P.I. & $\begin{array}{c}1 / 25 \\
\text { ECS+ level Ila } \\
40 \mathrm{~mm}\end{array}$ & $\begin{array}{c}145 \\
\text { (108 RAND-3D) }\end{array}$ & 4 & 14 & 2 & None & 5 & CRT & NED \\
\hline 8 & I.L. & $\begin{array}{l}\text { 1/21 (left side) } \\
\text { ECS- level lla } 4 \text { mm } \\
\text { 0/15 (right side) }\end{array}$ & $\begin{array}{c}370 \\
\text { (150 RAND-3D) }\end{array}$ & 3 & 13 & 0 & None & 5 & None & NED \\
\hline 9 & L.A. & $0 / 16$ & $\begin{array}{c}110 \\
\text { (60 RAND-3D) }\end{array}$ & 3 & 8 & 1 & None & 5 & CRT & NED \\
\hline 10 & F.R. & $\begin{array}{c}1 / 17 \\
\text { ECS- level II } 5 \mathrm{~mm}\end{array}$ & $\begin{array}{c}130 \\
\text { (100 RAND-3D) }\end{array}$ & 2 & 6 & 1 & None & 5 & RT & NED \\
\hline 11 & M.I. & $\begin{array}{c}7 / 21 \\
1 \text { ECS+ level Illa } \\
28 \mathrm{~mm} \\
3 \text { ECS- level III } 18 \mathrm{~mm} \\
3 \text { ECS- Level IV14 mm }\end{array}$ & $\begin{array}{c}45 \\
\text { (38 RAND-3D) }\end{array}$ & 2 & 4 & 1 & None & 5 & CRT & NED \\
\hline 12 & R.D. & $\begin{array}{c}1 / 27 \\
\text { ECS- level III } 11 \mathrm{~mm}\end{array}$ & $\begin{array}{c}120 \\
\text { (90 RAND-3D) }\end{array}$ & 5 & 12 & 0 & $\begin{array}{l}\text { Skin flap } \\
\text { partial } \\
\text { necrosis }\end{array}$ & 4 & RT & NED \\
\hline 13 & E.S. & $\begin{array}{c}\text { 2/32 } \\
1 \text { ECS+ level II } 6 \mathrm{~mm} \\
1 \mathrm{ECS}-\text { level III } 5 \mathrm{~mm}\end{array}$ & $\begin{array}{c}200 \\
\text { (130 RAND-3D) }\end{array}$ & 4 & 13 & 3 & None & 5 & CRT & NED \\
\hline 14 & C.A. & $\begin{array}{c}1 / 27 \\
\text { ECS- level lla } 18 \mathrm{~mm}\end{array}$ & $\begin{array}{c}158 \\
\text { (139 RAND-3D) }\end{array}$ & 5 & 8 & 0 & None & 5 & RT & NED \\
\hline 15 & N.M. & $\begin{array}{l}0 / 53 \\
\text { ECS- }\end{array}$ & $\begin{array}{c}120 \\
\text { (90 RAND-3D) }\end{array}$ & 3 & 4 & 3 & None & 5 & None & NED \\
\hline 16 & P.A. & - & $\begin{array}{c}85 \\
\text { (72 RAND-3D }\end{array}$ & 2 & 2 & 0 & None & 5 & CT & NED \\
\hline
\end{tabular}


The median follow-up period was 10 months and with no signs of loco-regional recurrence (no evidence of disease in any patient) (100\%).

In this group, the median satisfaction score was 4.9 of 5 (Fig. 4). Both surgeons were extremely satisfied with the outcomes (median surgeon satisfaction score was 5). None of the surgeons experienced side effects.

When dissecting level II, the percentage of surgery carried out under direct vision versus 3D exoscopic vision was calculated $(90 \%$ vs $10 \%)$. For level III, the percentage of 3D exoscopic vision rose to $20 \%$, in particular, when dissecting the lower part of this level. For levels IV-V, the rate of 3D exoscopic vision was about $20 \%$ when proceeding only via a retroauricular approach (4 patients did not require tracheostomy), while it was less than 5\% when proceeding via the median skin window. Dissection of level I was carried out completely under 3D exoscopic vision and, in our experience, this approach was particularly appropriate and precise.

\section{Group with thyroid malignant pathology}

There were 10 patients in this group ( 9 women, 1 man) with a median age of 45 years (range 28-72) (Tab. III). The average BMI was 23.4, with a maximum value of 28 . In this group, the Kocher skin incision was used to facilitate a straightforward and rapid dissection of levels IV and V. There were no intra-operative complications in patients in this group. Skin flap dehiscence was observed in only one patient (10\%) and was treated with a local dressing. Postsurgical pain was evaluated with a NRS (0 to 10$)^{6}$ and was on average 0.7 with a maximum value of 2 . All patients received $1 \mathrm{~g}$ paracetamol intravenously three times a day to relieve pain until discharge. None needed supplemental

Table III. Group with thyroid malignancies (continues on page 427).

\begin{tabular}{|c|c|c|c|c|c|c|c|c|c|}
\hline No. & ID & Age (yo) & Sex & BMI & Pre-treatment & Histology & Surgery & $\begin{array}{l}\text { CTNM } \\
\text { (TNM VIII } \\
\text { Eds) }\end{array}$ & $\begin{array}{l}\text { pTNM } \\
\text { (TNM VIII } \\
\text { Eds) }\end{array}$ \\
\hline 1 & S.G. & 43 & Female & 22 & None & Papillary carcinoma & $\begin{array}{c}\text { Total thyroidectomy + } \\
\text { ipsilateral SND } \\
\text { (II-V levels) }\end{array}$ & T1aN1b & T1aN1b \\
\hline 2 & R.J. & 44 & Female & 20 & None & Papillary carcinoma & $\begin{array}{c}T \\
\text { Total thyroidectomy + } \\
\text { ipsilateral SND } \\
\text { (II-V levels) }\end{array}$ & T1bN1b & T1bN1b \\
\hline 3 & C.M. & 45 & Female & 28 & None & Papillary carcinoma & $\begin{array}{l}\text { Total thyroidectomy + } \\
\text { ipsilateral SND } \\
\text { (II-V levels) }\end{array}$ & T2N1b & T2N1b \\
\hline 4 & S.R. & 72 & Male & 25 & None & Papillary carcinoma & $\begin{array}{l}\text { Total thyroidectomy + } \\
\text { ipsilateral SND (II-V levels) }\end{array}$ & T1N1b & $\mathrm{T} 1 \mathrm{Nb}$ \\
\hline 5 & F.F. & 33 & Female & 18 & None & Papillary carcinoma & $\begin{array}{l}\text { Total thyroidectomy + } \\
\text { ipsilateral SND (II-V levels) }\end{array}$ & T1aN1b & T1aN1b \\
\hline 6 & C.F. & 33 & Female & 28 & None & Papillary carcinoma & $\begin{array}{l}\text { Total thyroidectomy + } \\
\text { ipsilateral SND (II-V levels) }\end{array}$ & T1aN1b & T1aN1b \\
\hline 7 & M.F. & 28 & Female & 25 & None & Papillary carcinoma & $\begin{array}{l}\text { Total thyroidectomy + } \\
\text { ipsilateral SND (II-V levels) }\end{array}$ & T1aN1b & T1bN1b \\
\hline 8 & D.R.R. & 62 & Female & 20 & None & Medullary carcinoma & $\begin{array}{l}\text { Total thyroidectomy + } \\
\text { ipsilateral SND (II-IV levels) }\end{array}$ & T1No & T1bNo \\
\hline 9 & C.L. & 33 & Female & 25 & $\begin{array}{l}\text { Total } \\
\text { thyroidectomy }\end{array}$ & Papillary carcinoma & Ipsilateral SND (II-V levels) & TxN1b & TxN1b \\
\hline 10 & B.E. & 57 & Female & 23 & None & Medullary carcinoma & $\begin{array}{c}\text { Total thyroidectomy + } \\
\text { ipsilateral SND } \\
\text { (II-V levels) }\end{array}$ & T2N1b & T2N1b \\
\hline
\end{tabular}

ECS: extracapsular spread; NED: no evidence of disease; SND: selective neck dissection. "NRS: numerical rating scale where 0 is no pain and 10 is the worst pain imaginable. ${ }^{+}$Satisfaction score: 1, extremely dissatisfied; 2, dissatisfied; 3, average; 4, satisfied; 5, extremely satisfied. 


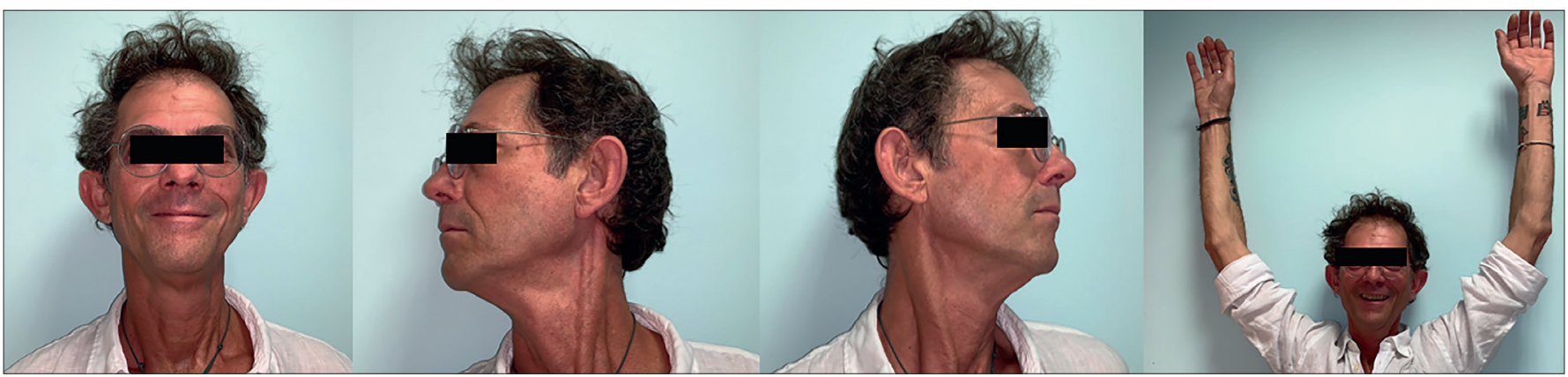

Figure 4. Cosmetic results in a patient treated for head neck malignant pathology (oropharyngeal squamous cell carcinoma).

therapy with NSAIDs or opioids and no patient required a blood transfusion. The number of resected lymph nodes ranged from 20 to 48 , with a mean of 36.5 nodes.
The average docking time to obtain an adequate operative setting was 6.5 minutes and the average intra-operative period was 88.1 minutes (60-120 minutes) (includ-

Table III. Group with thyroid malignancies (follows from page 426).

\begin{tabular}{|c|c|c|c|c|c|c|c|c|c|c|}
\hline No. & ID & $\begin{array}{l}\text { Lymph node } \\
\text { (positive/total) } \\
\text { (ECS, Level, } \\
\emptyset \text { max mm) }\end{array}$ & $\begin{array}{l}\text { Duration of } \\
\text { operation } \\
\text { (minutes) }\end{array}$ & $\begin{array}{l}\text { Drainage } \\
\text { period } \\
\text { (days) }\end{array}$ & $\begin{array}{l}\text { Hospitalisation } \\
\text { stay (days) }\end{array}$ & $\begin{array}{l}\text { Post- } \\
\text { operative } \\
\text { pain* } \\
\text { (NRS) }\end{array}$ & Complications & $\begin{array}{c}\text { Satisfaction } \\
\text { score }^{\dagger}\end{array}$ & $\begin{array}{l}\text { Adjuvant } \\
\text { treatment }\end{array}$ & $\begin{array}{l}\text { Follow- } \\
\text { up }\end{array}$ \\
\hline 1 & S.G. & $\begin{array}{c}5 / 48 \\
4 \text { ECS+ Level IV } \\
1 \text { ECS- Level III }\end{array}$ & $\begin{array}{c}180 \\
(120 \text { RAND-3D) }\end{array}$ & 2 & 3 & 2 & None & 5 & $I^{131}$ & NED \\
\hline 2 & R.J. & $\begin{array}{c}4 / 21 \\
\text { ECS- level II-III } 9 \text { mm }\end{array}$ & $\begin{array}{c}150 \\
\text { (90 RAND-3D) }\end{array}$ & 2 & 3 & 2 & None & 5 & $I^{131}$ & NED \\
\hline 3 & C.M. & $\begin{array}{l}\text { 7/20 } \\
\text { ECS- level II-III } \\
30 \mathrm{~mm}\end{array}$ & $\begin{array}{c}155 \\
\text { (95 RAND-3D) }\end{array}$ & 3 & 5 & 0 & None & 5 & $I^{131}$ & NED \\
\hline 4 & S.R. & $\begin{array}{c}3 / 47 \\
2 \text { ECS+ Level Ilb - V } \\
26 \mathrm{~mm}\end{array}$ & $\begin{array}{c}134 \\
(74 \text { RAND-3D) }\end{array}$ & 2 & 4 & 0 & $\begin{array}{l}\text { Shoulder } \\
\text { syndrome }\end{array}$ & 5 & $\left.\right|^{131}$ & NED \\
\hline 5 & F.F. & $\begin{array}{c}5 / 56 \\
2 \text { ECS- level II } 9 \mathrm{~mm} \\
3 \text { ECS- level IV } 5 \mathrm{~mm}\end{array}$ & $\begin{array}{c}140 \\
\text { (80 RAND-3D) }\end{array}$ & 2 & 3 & 1 & $\begin{array}{l}\text { Skin flap } \\
\text { dehiscence }\end{array}$ & 4 & $I^{131}$ & NED \\
\hline 6 & C.F. & $\begin{array}{c}3 / 37 \\
\text { ECS- level III-IV } \\
40 \mathrm{~mm}\end{array}$ & $\begin{array}{c}175 \\
\text { (93 RAND-3D) }\end{array}$ & 2 & 3 & 0 & None & 5 & $I^{131}$ & NED \\
\hline 7 & M.F. & $\begin{array}{c}6 / 32 \\
2 \text { ECS+ level IV } 24 \text { mm } \\
2 \text { ECS- level Ila } 12 \mathrm{~mm} \\
2 \text { ECS- level III } 9 \mathrm{~mm}\end{array}$ & $\begin{array}{c}169 \\
\text { (103 RAND-3D) }\end{array}$ & 3 & 4 & 0 & None & 5 & $f^{131}$ & NED \\
\hline 8 & D.R.R. & $0 / 25$ & $\begin{array}{c}135 \\
\text { (76 RAND-3D) }\end{array}$ & 2 & 3 & 2 & None & 5 & None & NED \\
\hline 9 & C.L. & $\begin{array}{c}1 / 42 \\
\text { ECS- level IV } 10 \mathrm{~mm}\end{array}$ & 60 RAND-3D & 2 & 2 & 0 & None & 4 & $\left.\right|^{131}$ & NED \\
\hline 10 & B.E. & $\begin{array}{c}1 / 37 \\
\text { ECS- level IV 0,8 mm }\end{array}$ & $\begin{array}{c}150 \\
\text { (90 RAND-3D) }\end{array}$ & 2 & 3 & 0 & None & 5 & None & NED \\
\hline
\end{tabular}




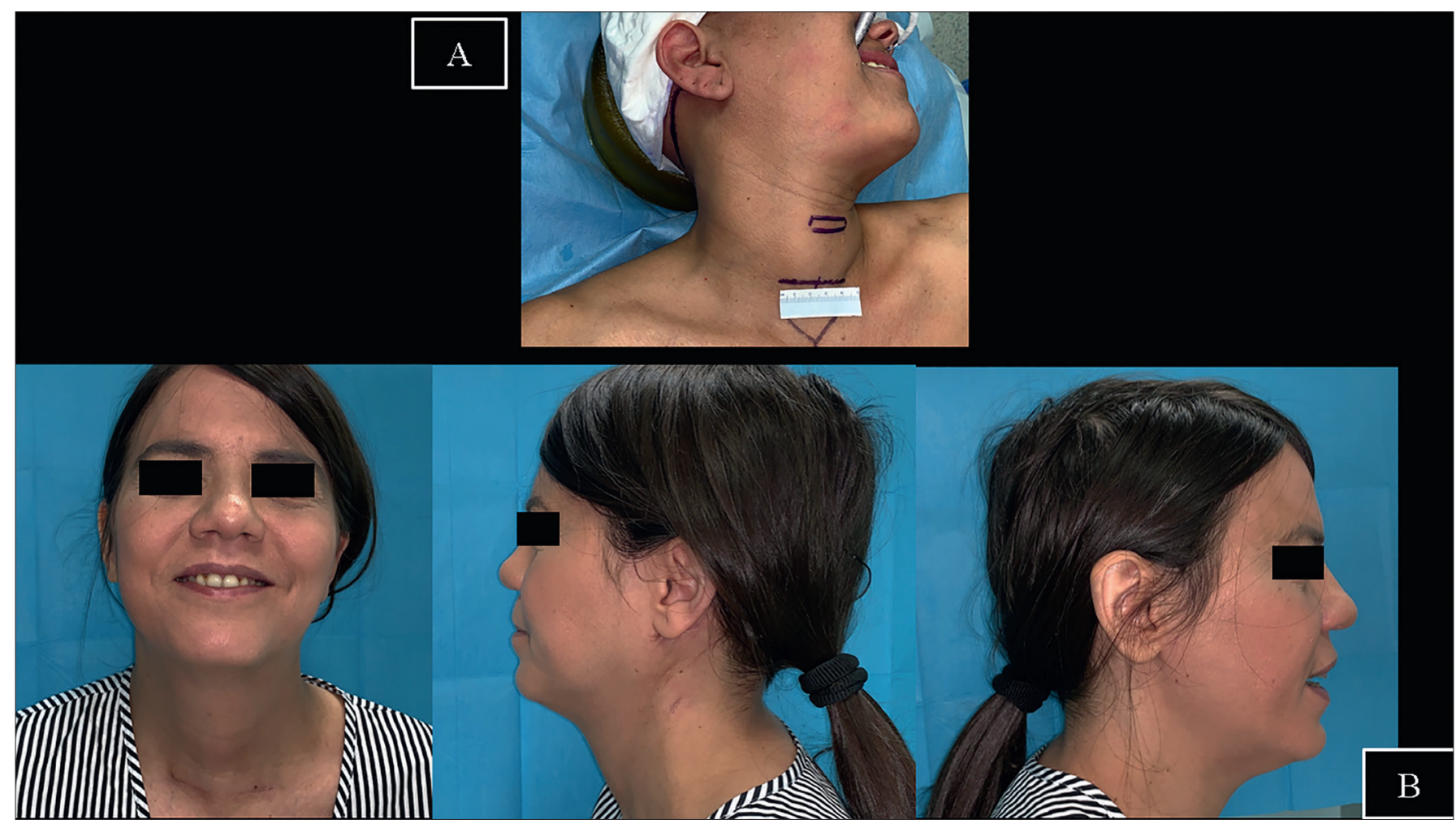

Figure 5. (A) Retroauricular facelift skin incision and cervical median skin window. (B) Cosmetic results in a patient treated for thyroid malignant pathology.

ing flap harvesting, placement of retractor, dissection). The average duration of drainage was 3.33 days and the average length of hospitalisation was 3.3 days. There were no other post-operative neurologic deficits (marginalis mandibulae nerve paralysis). One patient (10\%) experienced shoulder syndrome, and in another patient, we observed partial skin flap necrosis which was treated with a dressing. The median follow-up period was 6 months with no signs of locoregional recurrence. In this group, al patients were satisfied with their cosmetic outcome (median satisfaction score 4.8 of 5) (Fig. 5). Both surgeons were extremely satisfied with the outcomes (median surgeon satisfaction score was 5). None of the surgeons experienced side effects. To dissect every cervical level in this group, the rates of 3D exoscopic vision were similar to those found in the group with head and neck malignancy.

\section{Discussion}

At the beginning of the 1980s, there was a gradual and progressive adoption of minimally-invasive techniques, initially in abdominal, gynaecological, urological and thoracic surgical procedures, and more recently in head/neck surgery. With the advent of endoscopic techniques and subsequent robotic ones, the need arose for remote access to the neck through a retroauricular approach with the goal of improving surgical accuracy, and functional and aesthetic outcomes, while ensuring the same oncological outcomes ${ }^{3,4,7}$. Use of a retroauricular approach for parotidectomy, originally presented in 1994 by Terris et al. ${ }^{8}$, delivers a short and direct route to the neck, requiring minimal dissection and providing an adequate workspace.

In 1996, Gagner ${ }^{9}$ described the first endoscopically assisted operation (subtotal parathyroidectomy) with a retroauricular skin incision. More recently, several authors ${ }^{10-15}$ have described their initial experiences using the retroauricular endoscope-assisted approach for different head and neck operations such as supraomohyoid neck dissections (SOHND), benign neck mass excision, submandibular gland excision and thyroidectomy. All authors agree that the endoscopic approach to the neck by retroauricular skin incision is feasible, safe, and oncologically effective, and can be used in selected cases with a clear cosmetic benefit, even without using the da Vinci robotic system.

Several limitations and concerns with regards to the facelift approach for endoscope-assisted surgical procedures have prevented its widespread adoption. One of the main disadvantages is that, although angled optics are available, rigid endoscopic instruments may have limitations in approaching the site of dissection, especially in narrow and angled 
working spaces such as the neck. The endoscopic view may be hindered by surrounding tissue or by the instruments and it is only a two-dimensional view with lack of depth perception when compared with the three-dimensional image provided by robotic imaging systems or by the VITOM-3D system. To date, 3D endoscopes are available but no studies have been published on their use in neck dissection. Moreover, the endoscope and instruments are controlled by two surgeons and, despite the availability of endoscope-holders, they frequently encroach on each other's workspace making the operation somewhat problematic. The rigid, straight nature of the endoscope and other instruments and the lack of a third arm, available in robotic techniques, further contribute to the limited ability to manipulate them with a minimal tactile sensation. Finally, surgeons require time to develop a good skill level in handling the endoscope; the learning curve is time consuming and difficult ${ }^{10-15}$.

Regarding robotic-assisted neck dissection via a modified facelift or retroauricular approach, many advantages have been highlighted: stable three-dimensional binocular magnification ${ }^{16-20}$, motion scaling ${ }^{17,19}$, tremor filtration ${ }^{16-18,20}$, a shortened learning curve ${ }^{17-20}$ and superior surgeon ergonomics ${ }^{17,20,21}$.

The major disadvantage of robot-assisted neck dissection is the prolonged operation time compared to that of conventional neck dissection. In 2014, Kim et al. ${ }^{21}$ compared conventional neck dissection with robot-assisted neck dissection (RAND) and noted that the mean operative time for RAND group was significantly longer than that of the conventional neck dissection group, with no significant difference between the two groups in the mean number of lymph nodes retrieved. They concluded that therapeutic RAND via a retroauricular approach was successful with satisfactory aesthetic results in patients with node-positive head and neck cancer.

In addition, the learning curve is more complex compared with conventional neck dissection. Several authors recommend that robot-assisted neck dissection should be performed only by surgeons who have good experience with conventional neck dissection and using the surgical robot and who have been specifically trained in RAND ${ }^{21,22}$.

Undoubtedly, robot-assisted surgery is expensive, and this aspect represents one of the main obstacles for popularisation of this surgical option. With regard to costs, Yoo et al. ${ }^{23}$ reported that the mean cost of endoscopic thyroidectomy was $\$ 829$, or eight times less expensive than robotic thyroidectomy. Furthermore, not all institutions have da Vinci systems, there is often competition to use the robotic platform, and most hospitals, particularly in developing countries, cannot afford to purchase such an expensive device. The recent advent of 3D exoscopic surgery has allowed in- teresting technical improvements to be introduced in head and neck surgery, with technical solutions also applicable to neck dissection, with the aim of enhancing surgical vision through remote access, and minimising the costs of the procedure in comparison to robotic surgery. Based on these considerations, we recently carried out a preclinical investigation in the cadaver lab, focused on approaching conventional neck dissection using a retroauricular skin incision, and evaluating the applications and usefulness of the Storz 3D Exoscopic System during different stages of the surgical procedure. The acronym RAND-3D (3D exoscopic surgery) was coined to describe the use of this optical tool to perform neck dissection ${ }^{5}$.

Following the suggestions of one of the reviewers of that paper, we decided to change the acronym from RAND-3D to RANS-3D (VITOM-3D assisted retroauricular neck surgery) to avoid any possible confusion with another surgical technique, RAND (robotic-assisted neck dissection).

For dissection, we decided to use the VITOM-3D surgical system in combination with direct vision to obtain an adequate surgical view together with appropriate instrumentation to ensure oncologic safety and to prevent possible injury to neurovascular structures. Although our limited cases series and short follow-up period did not allow us to confirm the oncological effectiveness of this method, the number of lymph nodes retrieved in our sample was similar to those published in earlier studies with classic or retroauricular SOHND ${ }^{17,18,21}$.

In our experience, VITOM-3D allows the same vision to be shared among surgeons through a narrow skin window during the entire procedure. The authors suggest placing a polarised lens over the loupes, allowing a comfortable optical solution to be reached and continuous comparison between exoscope and loupe.

In the group with benign pathology in our study, the exoscope is particularly useful to the first surgeon to improve identification of the terminal branches of the facial nerve, and to the second surgeon during haemostatic manoeuvres. In the group with malignant pathology, performing SOHND to dissect levels II and III, some haemostatic manoeuvres, carried out by the second surgeon around the hypoglossal nerve and thyrolinguofacial trunk, can benefit from exoscopic vision. The percentage of $3 \mathrm{D}$ exoscopic vision rose to $20 \%$, in particular when dissecting the lower part of level III, indicating that the accuracy of the first surgeon had improved.

For the dissection of levels IV and V, the first surgeon moves to the head of the patient. This is undoubtedly the most challenging step in the operation because, at level IV, the working space is extremely narrow and the risk of an inadvertent injury to the internal jugular vein (IJV) or thorac- 
ic duct is high. Also during this step, the exoscope is useful to share the same good vision between both surgeons; however, the long working distance makes this dissection lengthy and difficult to manage, even for expert surgeons. To facilitate dissection of level IV and dramatically reduce the risks of vascular/lymphatic injury, access through the incision used to perform the tracheostomy is extremely valuable. The concept is to create a service skin window allowing the lateral margin of the sternocleidomastoid muscle, the upper belly of the omohyoid muscle, and the cervical vascular-nervous axis to be identified and then dissecting this level upward, reaching level III. In this group with malignant pathology, 12 patients underwent surgical tracheostomy at the beginning of the procedure, reducing the operating time for dissection of levels IV and the lower part of level V. Similarly, in the thyroid pathology group, with the Kocher skin incision currently used in thyroid surgery, dissection of levels IV and V was carried out without a significant increase in intraoperative time and allowing an effective surgical procedure while leaving only a small visible scar. To dissect levels IV and V, the rate of 3D exoscopic vision is about $20 \%$ when using only a retroauricular approach, while it is less than $5 \%$ when proceeding via the median skin window.

$3 \mathrm{D}$ visualisation was essential during level I dissection to improve identification of every anatomic structure. Considering the working distance and the presence of blood vessels on the muscular surfaces, it can be very useful to perform this dissection using a $24 \mathrm{~cm}$ LigaSure Maryland forceps, in haemostatic mode.

Technically feasible, this technique ensures a complete compartment-oriented dissection. Undoubtedly, the need for a second skin incision could underpower the good aesthetic results of the approach, but it allows the safe dissection of levels IV and V.

The characteristics of the VITOM-3D images are very similar to those obtained with the 3D optics of the da Vinci surgical platform, with its excellent ability to provide 3D visual information which is used to interactively control the exoscope camera. Other advantages of the VITOM-3D surgical system are the depth of field, magnification, image contrast and colour, allowing direct manipulation of the images of anatomic structures and magnification of anatomic details, for example vascularisation.

The system is comfortable to use for the surgeons as they remain in a sitting position for long periods with the screen in front of them at eye level: surgery carried out facing a 3D screen is not bothersome for operators, even for longer procedures.

Furthermore, the 3D exoscope provides the benefit of great utility in the learning process, especially for residents, fel- lows, students, and operating room (OR) staff, thanks to the same shared visual experience being available to each operator, and always with wide high-resolution monitors. Both images and video sequences can be stored in high definition, enabling surgeons to share videos in didactic sessions, meetings and surgical technique courses.

The current drawbacks are represented by the mechanical holder arm which is not always comfortable to move during surgery (a robotic holder has recently been introduced) and the necessity to wear 3D glasses for a prolonged period which can lead to headaches in some cases. In our experience, none of the surgeons experienced side effects.

Regarding the learning curve, we recently carried out a preclinical investigation in the cadaver lab focused on approaching conventional neck dissection using a retroauricular skin incision, and evaluated the applications and usefulness of the Storz 3D Exoscopic System during different stages of the surgical procedure ${ }^{5}$. Four human cadavers were obtained from the Italian Academy of Anatomic Dissection (AIAD). Dissection was carried out bilaterally on each cadaver by two senior head and neck surgeons (E.C., G.S.), with good expertise in head neck open and endoscopic approaches, the same surgeons who carried out the surgical procedures in this study. In our opinion, for a surgeon with good experience in open and endoscopic surgery, the learning curve is extremely fast and straightforward.

Regarding the economic aspects, the entire cost of the exoscopic platform is similar to that of an operating microscope with an electromagnetic brake (about $€ 110,000$ ). The cost of disposables for each surgical procedure is about $€ 40$, composed of two sterile sheaths for the holder and controller chamber; even the cost of maintenance is considerably lower than robotic machine. Much of the same platform can be used daily in endoscopic surgery of the upper aerodigestive tract, the most frequently performed endoscopic procedure, and this contributes greatly to the amortisation of costs.

\section{Conclusions}

The current study indicates that RANS-3D is an interesting surgical approach for neck surgery. The hybrid execution of neck dissection under direct and exoscopic vision represents a valid alternative to video-assisted endoscopic- and robot-assisted techniques. As with any new technology, there is a learning curve and a period of adaptation to overcome. In benign pathologies, the exoscope is particularly useful to improve the precision of dissection, especially during haemostatic manoeuvres in parotid surgery. According to the experience gained with this cohort, most cervical levels (especially levels II and III) can be 
dissected under direct visualisation with the retroauricular approach. Exoscopic assistance is essential for level I dissection. However, dissection of levels IV and V exclusively through a retroauricular skin incision is quite unsafe using this technique because the working space is extremely narrow. This is the reason why we suggest a median skin "window", which gives better vision and greater safety in the dissection of vascular and lymphatic structures. The surgical morbidity and oncologic validation of the procedure should be verified with further prospective clinical studies and with longer follow-up periods, focused on the direct comparison between conventional open, robotic and exoscopic techniques. The level of patient satisfaction with cosmetic outcome is very high, encouraging us to continue with this fascinating cervical approach.

\section{References}

1 Muenscher A, Dalchow C, Kutta H, et al. The endoscopic approach to the neck: a review of the literature, and overview of the various techniques. R Surg Endosc 2011;25:1358-1363. https://doi.org/10.1007/ s00464-010-1452-9

2 Lang BH, Wong CK, Tsang JS, et al. A systematic review and metaanalysis comparing outcomes between robotic-assisted thyroidectomy and non-robotic endoscopic thyroidectomy. Surg Res 2014;191:389398. https://doi.org/10.1016/j.jss.2014.04.023

3 Kim WS, Lee HS, Kang SM, et al. Feasibility of robot-assisted neck dissections via a transaxillary and retroauricular ("TARA") approach in head and neck cancer: preliminary results. Ann Surg Oncol 2012;19:1009-1017. https://doi.org/10.1245/s10434-011-2116-2

4 Kang SW, Lee SH, Ryu HR, et al. Initial experience with robot-assisted modified radical neck dissection for the management of thyroid carcinoma with lateral neck node metastasis. Surgery 2010;148:12141221. https://doi.org/10.1016/j.surg.2010.09.016

5 Crosetti E, Arrigoni G, Manca A, et al. VITOM-3D assisted neck dissection via a retroauricular approach (RAND-3D): a preclinical investigation in a cadaver lab. Acta Otorhinolaryngol Ital 2020;40:343-351. https://doi.org/10.14639/0392-100X-N0757

6 Delgado DA, Lambert BS, Boutris N, et al. Validation of digital visual analog scale pain scoring with a traditional paper-based visual analog scale in adults. J Am Acad Orthop Surg Glob Res Rev 2018;2:e088. https://doi.org/10.5435/JAAOSGlobal-D-17-00088

7 Lee HS, Kim WS, Hong HJ, et al. Robot-assisted supraomohyoid neck dissection via a modified face-lift or retroauricular approach in earlystage $\mathrm{cN} 0$ squamous cell carcinoma of the oral cavity: a comparative study with conventional technique. Ann Surg Oncol 2012;19:38713878. https://doi.org/10.1245/s10434-012-2423-2

8 Terris DJ, Tuffo KM, Fee WE. Modified facelift incision for parotidectomy. J Laryngol Otol 1994;108:574-578. https://doi.org/10.1017/ s002221510012746x
9 Gagner M. Endoscopic subtotal parathyroidectomy in patients with primary hyperparathyroidism. Br J Surg 1996;83:875. https://doi. org/10.1002/bjs. 1800830656

10 Baek CH, Jeong HS. Endoscope-assisted submandibular sialadenectomy: a new minimally invasive approach to the submandibular gland. Am J Otolaryngol 2006;27:306-309. https://doi.org/10.1016/j.amjoto.2005.11.018

11 Chen MK, Su CC, Tsai YL, et al. Minimally invasive endoscopic resection of the submandibular gland: a new approach. Head Neck 2006;28:1014-1017. https://doi.org/10.1002/hed.20469

12 Guyot L, Duroure F, Richard O, et al. Submandibular gland endoscopic resection: a cadaveric study. Int J Oral Maxillofac Surg 2005;34:407-410. https://doi.org/10.1016/j.ijom.2004.11.001

13 Kessler P, Bloch-Birkholz A, Birkholz T, et al. Feasibility of an endoscopic approach to the submandibular neck region - experimental and clinical results. Br J Oral Maxillofac Surg 2006;44:103-106. https:// doi.org/10.1016/j.bjoms.2005.03.016

14 Byeon HK, Holsinger FC, Koh YW, et al. Endoscopic supraomohyoid neck dissection via a retroauricular or modified facelift approach: preliminary results. Head Neck 2014;36:425-430. https://doi. org/10.1002/hed.23308

15 Song CM, Jung YH, Sung MW, et al. Endoscopic resection of the submandibular gland via a hairline incision: a new surgical approach. Laryngoscope 2010;120:970-974. https://doi.org/10.1002/lary.20865

16 Goh HK, Ng YH, Teo DT. Minimally invasive surgery for head and neck cancer. Lancet Oncol 2010;11:281-286. https://doi.org/10.1016/ S1470-2045(09)70379-1

17 Kim WS, Byeon HK, Park YM, et al. Therapeutic robot-assisted neck dissection via a retroauricular or modified facelift approach in head and neck cancer: a comparative study with conventional transcervical neck dissection. Head Neck 2015;37:249-254. https://doi. org/10.1002/hed.23595

18 Byeon HK, Holsinger FC, Kim DH, et al. Feasibility of robotassisted neck dissection followed by transoral robotic surgery. $\mathrm{Br}$ J Oral Maxillofac Surg 2015;53:68-73. https://doi.org/10.1016/j. bjoms.2014.09.024

19 Blanco RG, Boahene K. Robotic-assisted skull base surgery: preclinical study. J Laparoendosc Adv Surg Tech A 2013;23:776-782. https:// doi.org/10.1089/lap.2012.0573

20 Lee HS, Kim D, Lee SY, et al. Robot-assisted versus endoscopic submandibular gland resection via retroauricular approach: a prospective nonrandomized study. Br J Oral Maxillofac Surg 2014;52:179-184. https://doi.org/10.1016/j.bjoms.2013.11.002

21 Kim WS, Ban MJ, Chang JW, et al. Learning curve for robot-assisted neck dissection in head and neck cancer: a 3-year prospective case study and analysis. JAMA Otolaryngol Head Neck Surg 2014;140:1191-1197. https://doi.org/10.1001/jamaoto.2014.2830

22 Albergotti WG, Byrd JK, Nance M, et al. Robot-assisted neck dissection through a modified facelift incision. Ann Otol Rhinol Laryngol 2016;125:123-129. https://doi.org/10.1177/0003489415601127

23 Yoo H, Chae BJ, Park HS, et al. Comparison of surgical outcomes between endoscopic and robotic thyroidectomy. J Surg Oncol 2012;105:705-708. https://doi.org/10.1002/jso.22106 